\title{
A Case of Advanced Hepatocellular Carcinoma with Partial Response after Continuous Ramucirumab Treatment beyond Radiological Progression
}

\author{
Yoshiyuki Wada Yuko Takami Tomoki Ryu Hiroki Ureshino \\ Hajime Imamura Shin Sasaki Hideki Saitsu
}

Department of Hepato-Biliary-Pancreatic Surgery, Clinical Research Institute, National

Hospital Organization Kyushu Medical Center, Fukuoka, Japan

\author{
Keywords \\ Ramucirumab · Hepatocellular carcinoma · Liver · Malignancy · Response · Progressive \\ disease
}

\section{Abstract}

A 73-year-old male was initially treated with sorafenib for advanced stage HCC. However, the disease progressed 2 months after starting sorafenib. Progressive disease (PD) was confirmed by radiological examination, which revealed mediastinal and abdominal lymph node metastasis, pulmonary metastasis, and intrahepatic recurrence. The patient was enrolled in the REACH-2 (NCT02435433) trial and randomized to receive ramucirumab $(8 \mathrm{mg} / \mathrm{kg}$ div. every 2 weeks). The patient had a Child-Pugh score of $6 \mathrm{~A}$ and his AFP level was found to be 1,256.8 $\mathrm{ng} / \mathrm{mL}$ at initiation. Radiological examination revealed PD, 5 months after starting ramucirumab. Ramucirumab treatment was continued after the confirmation of radiological PD, not but clinical progression, as allowed by the study protocol. His AFP level increased after continuous ramucirumab treatment, however, it suddenly decreased from 7,653 ng/mL to within normal limits 10 months after initiation of ramucirumab treatment. Radiological evaluation revealed a significant decrease in the size of the tumors, which constituted a partial response (PR). We reported a rare case of advanced HCC with PR to a continuous ramucirumab treatment after radiological $P D$. 
Wada et al.: A PR Case of HCC under Ramucirumab beyond PD

\section{Introduction}

Systemic chemotherapy for unresectable hepatocellular carcinoma (HCC) has rapidly developed recently. Tyrosine-kinase inhibitors, such as sorafenib [1,2], regorafenib [3], and lenvatinib [4], are available as agents for the treatment of unresectable HCC. In 2018, ramucirumab [5] and cabozantinib [6] were demonstrated to have an improved overall survival as second-line therapies for patients with unresectable HCC refractory or intolerable to sorafenib.

Ramucirumab, a human IgG1 monoclonal antibody that inhibits ligand activation of vascular endothelial growth factor receptor-2, showed significant improvement of overall survival in unresectable HCC patients with a baseline $\alpha$-fetoprotein (AFP) concentration $\geq 400$ $\mathrm{ng} / \mathrm{mL}$ after intolerance to, or progression during, the previous sorafenib therapy in a phase 3 trial (REACH-2 trial) [5]. However, the clinical features of ramucirumab remain unclear in clinical practice. Here, we report a very rare case of unresectable HCC that showed a partial response (PR) after continuous ramucirumab treatment, beyond confirmation of radiological progressive disease (PD).

\section{Case Report}

A 71-year-old Japanese male with chronic hepatitis C was referred to our hospital for hepatic tumors. His radiological examination revealed HCC with 3 lesions with a maximum diameter of $2.1 \mathrm{~cm}$ (Barcelona-Clinic Liver Cancer (BCLC) stage A). He underwent surgical microwave ablation therapy with a complete treatment response in 2012. Two years later, in 2014, 5 intrahepatic recurrences and 2 pulmonary metastases were detected, and the patient was again treated with surgical microwave ablation therapy and video-assisted thoracic surgery (VATS). Moreover, one and half years later, the patient underwent VATS for solitary pulmonary metastasis. In 2016 (the patient was then 74 years old), a computed tomography (CT) scan revealed mediastinal lymph node metastasis and intrahepatic recurrence. The patient was administered sorafenib at a reduced dose of $400 \mathrm{mg}$ daily to prevent treatment withdrawal at an early period. At the initiation of sorafenib, he had a Child-Pugh score of 5A, performance status 0. His serum AFP level was high $(261.5 \mathrm{ng} / \mathrm{mL})$. However, after the administration of sorafenib, as the serum AFP level increased to $951 \mathrm{ng} / \mathrm{mL}$, and the first radiological estimation showed progression of the mediastinal lymph node and new lung metastasis, the patient was assessed by the RECIST 1.1 and modified RECIST criteria to have PD. Thus, the patient was enrolled in a randomized double-blind trial (REACH-2 study; NCT02435433).

\section{Results}

The patient was randomized to receive ramucirumab. At the initiation of ramucirumab treatment, he had a Child-Pugh score of $6 \mathrm{~A}$ (albumin $3.2 \mathrm{~g} / \mathrm{dL}$, total bilirubin $0.5 \mathrm{mg} / \mathrm{dL}$, prothrombin time 72\%). Physical examination showed a height of $164 \mathrm{~cm}$, weight $53.8 \mathrm{~kg}$, and performance status 0. His serum AFP level was increased to 1,256.8 ng/mL. A CT scan showed progression of mediastinal lymph node metastasis with a short axis of $25 \mathrm{~mm}$ (Fig. 1a), 2 intrahepatic recurrences with a diameter of $9 \mathrm{~mm}$, and a new development of pulmonary metastasis with a diameter of $7 \mathrm{~mm}$ (Fig. 2a). The patient was treated with ramucirumab $8 \mathrm{mg} / \mathrm{kg}$ div. every 2 weeks, according to the study protocol of the REACH- 2 trial.

Five months after the administration of ramucirumab, radiological examination revealed progression of mediastinal lymph node metastasis (Fig. 1b) and pulmonary metastasis (Fig. 2b). Moreover, there was an abdominal lymph node metastasis. Transient grade 2 edema 

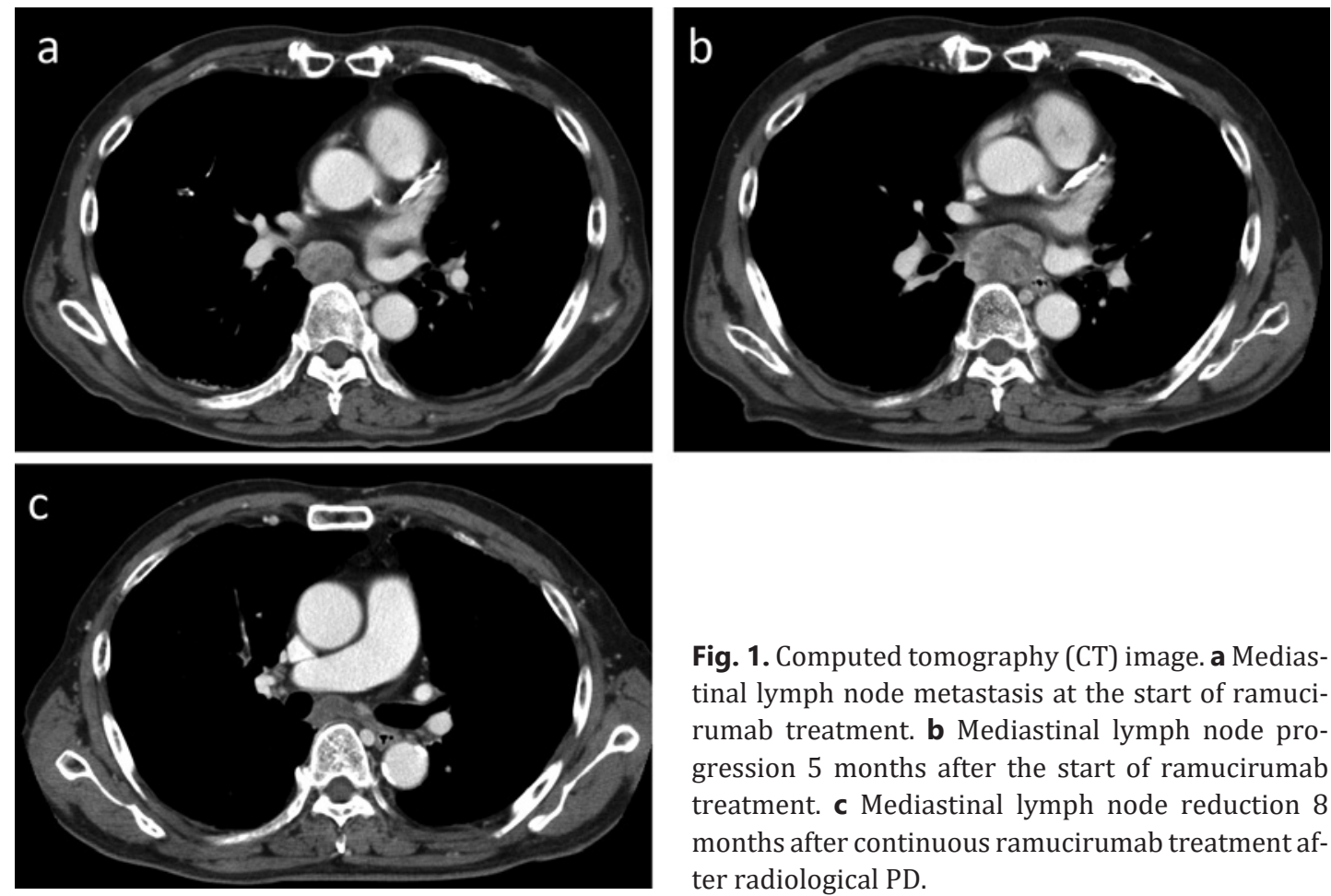

Fig. 1. Computed tomography (CT) image. a Mediastinal lymph node metastasis at the start of ramucirumab treatment. b Mediastinal lymph node progression 5 months after the start of ramucirumab treatment. c Mediastinal lymph node reduction 8 months after continuous ramucirumab treatment after radiological PD.
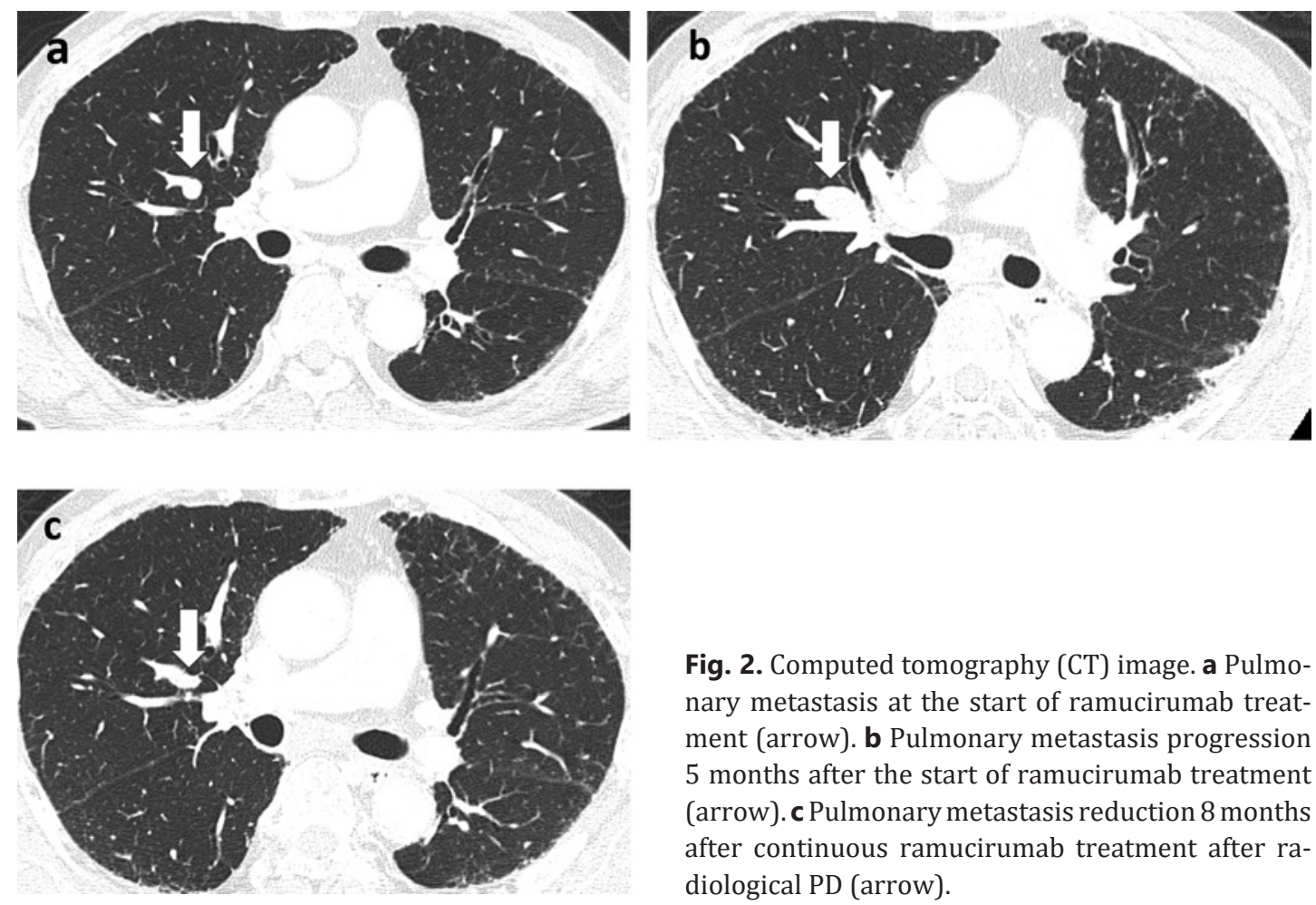

Fig. 2. Computed tomography (CT) image. a Pulmonary metastasis at the start of ramucirumab treatment (arrow). b Pulmonary metastasis progression 5 months after the start of ramucirumab treatment (arrow).c Pulmonary metastasis reduction 8 months after continuous ramucirumab treatment after radiological PD (arrow). 


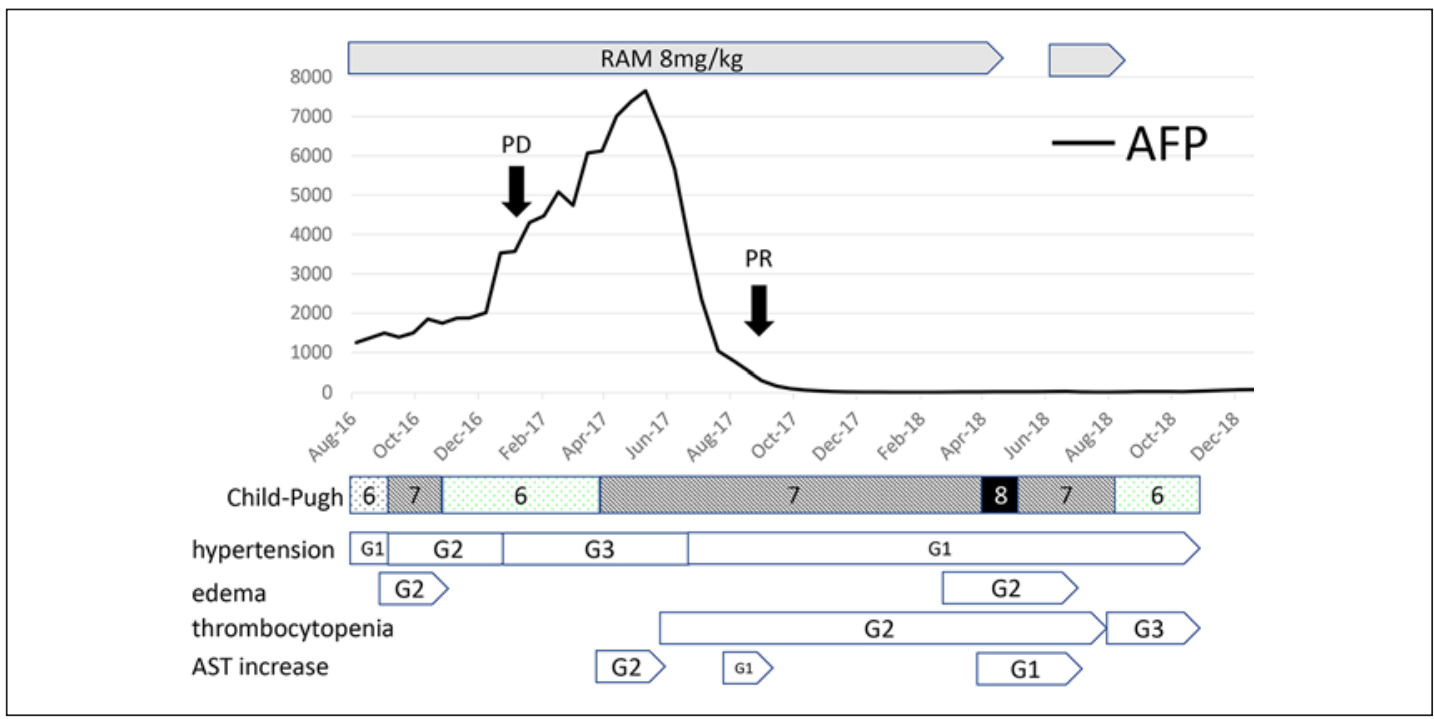

Fig. 3. Clinical course of ramucirumab (RAM) treatment and change in the patient's $\alpha$-fetoprotein (AFP) levels. G, grade; AST, aspartate aminotransferase.

and grade 2 hypertension were confirmed as adverse events during treatment until radiological PD (Fig. 3). At the time of confirmation of radiological PD, the adverse events were tolerable, and the liver function was preserved at a Child-Pugh score of $6 \mathrm{~A}$.

The study protocol stated that if the patient experienced a treatment benefit in the opinion of the investigator, the patient could continue the study treatment beyond radiographic progression until clinical progression. Furthermore, the determination of clinical progression was at the discretion of the investigator and would include both objective and subjective data. In accordance with the study protocol of the REACH-2 study, the patient was continued on ramucirumab treatment even after confirmation of radiological PD. After continuous treatment after PD, hypertension worsened to grade 3 , which was controlled by the addition of an antihypertensive agent. His serum AFP level increased after continuous ramucirumab treatment, although radiological estimation assessed stable disease. However, the patient's serum AFP level suddenly decreased from 7,653 ng/mL to within normal limits 10 months after initiation of ramucirumab treatment (Fig. 3).

Radiological evaluation revealed a significant decrease of mediastinal metastasis (Fig. 1c) and pulmonary metastasis (Fig. 2c), which was defined as a PR by RECIST v1.1 criteria. While on continuous ramucirumab treatment, tolerable adverse events including hypertension, edema, and the increase in aspartate aminotransferase levels were controlled well. However, the treatment was terminated because of uncontrollable grade 3 thrombocytopenia, 26 months after the initiation of ramucirumab. At the time of termination of treatment, radiological evaluation confirmed the preservation of PR. The patient was then treated with lenvatinib for 6 months. The patient died 13 months after termination of ramucirumab treatment.

\section{Discussion}

Ramucirumab demonstrated a significant improvement in the overall survival in advanced HCC patients with serum level $\geq 400 \mathrm{ng} / \mathrm{mL}$ after sorafenib in the REACH-2 trial [5], despite a low objective response rate of $4 \%$. Here, we describe a very rare case of a late- 
occurring and long-lasting PR effect after continuous ramucirumab treatment even after the confirmation of radiological PD. This case contains clinically important information on (1) continuous ramucirumab treatment after radiological PD and (2) sustained PR after continuous ramucirumab treatment after radiological PD.

First, it remains unclear whether continuous ramucirumab treatment is effective after radiological PD. However, previous studies have reported the efficacy of continuous sorafenib treatment after radiological PD [7,8]. In particular, continuous sorafenib treatment was effective for patients with thrombotic thrombocytopenia purpura $>4$ months, the so-called "slow PD" patients [8,9]. This case study reports a radiological PD, 5 months after starting ramucirumab, and we considered this as "slow PD" as well. Furthermore, the study protocol allowed continuation of treatment beyond RECIST-based PD, provided there was clinical benefit in the opinion of the investigator.

High tolerability of ramucirumab during treatment adds to its clinical benefits. It is desirable to confirm the efficacy of continuous ramucirumab treatment after radiological PD in the future, as it may be adapted as a treatment alternative for patients with slow PD.

Second, a clear reason for the sustained PR after continuous ramucirumab treatment even after radiological PD remains obscure. An interesting mechanism was reported for significant activation of VEGF-signaling in AFP-high tumors [10]. The competition of VEGF-A with the other ligands, such as VEGF-B or PGF, could favor its binding to VEGFR2 in AFP-high tumors, thus leading to its subsequent activation of VEGF signaling. As a result, ramucirumab may demonstrate a high antitumor activity in AFP-high tumors. In the reported case, an increased AFP level may have caused VEGFR-signaling activity by favoring VEGFR2, resulting in improved sensitivity toward ramucirumab during continuous treatment after radiological PD. Furthermore, this mechanism was supported by the results of the REACH-2 study which showed that a higher AFP level is associated with higher improvement of overall survival [5]. It is necessary to confirm whether a higher response to ramucirumab induced by a higher AFP level may result in the improvement of clinical outcomes in the future.

Next, it is very interesting to note the gradual worsening of hypertension in this case until the confirmation of radiological PR. Gradual worsening of hypertension may indicate that the response to ramucirumab was reinforced. It has been reported that hypertension as an adverse event during sorafenib treatment is correlated with improvement in progressionfree survival [11,12] or overall survival [11-13]. Regarding ramucirumab treatment, hypertension as an adverse event may be correlated to the response to ramucirumab.

This study has its limitations. In principle, treatment is usually changed to another available drug after PD. However; we can consider continuous use after PD in case that drug is the best option for the patient based on some reason (e.g., tolerability or availability).

In conclusion, we reported a rare case of advanced HCC with PR to a continuous ramucirumab treatment even after radiological PD. Regular use of ramucirumab as a continuous treatment regimen in advanced HCC warrants further investigation to characterize the clinical features of the drug and the patient population which will profit most.

\section{Statement of Ethics}

The patient gave informed consent to publish his case.

\section{Disclosure Statement}

The authors have no conflicts of interest to declare. 


\section{Funding Sources}

There was no funding provided for the writing of this paper.

\section{Author Contributions}

All authors contributed equally to the paper, including writing and revising it. All authors read and approved the final manuscript.

\section{References}

1 Llovet JM, Ricci S, Mazzaferro V, Hilgard P, Gane E, Blanc JF, et al.; Group SIS. Sorafenib in advanced hepatocellular carcinoma. N Engl J Med. 2008 Jul 24;359(4):378-90.

2 Cheng A-L, Kang Y-K, Chen Z, Tsao C-J, Qin S, Kim JS, et al. Efficacy and safety of sorafenib in patients in the Asia-Pacific region with advanced hepatocellular carcinoma: a phase III randomised, double-blind, placebocontrolled trial. Lancet Oncol. 2009;10(1):25-34.

3 Bruix J, Qin S, Merle P, Granito A, Huang YH, Bodoky G, et al. Regorafenib for patients with hepatocellular carcinoma who progressed on sorafenib treatment (RESORCE): a randomised, double-blind, placebocontrolled, phase 3 trial. Lancet. 2017 Jan 7;389(10064):56-66.

4 Kudo M, Finn RS, Qin S, Han KH, Ikeda K, Piscaglia F, et al. Lenvatinib versus sorafenib in first-line treatment of patients with unresectable hepatocellular carcinoma: a randomised phase 3 non-inferiority trial. Lancet. 2018 Mar 24;391(10126):1163-73.

5 Zhu AX, Kang YK, Yen CJ, Finn RS, Galle PR, Llovet JM, et al. Ramucirumab after sorafenib in patients with advanced hepatocellular carcinoma and increased alpha-fetoprotein concentrations (REACH-2): arandomised, double-blind, placebo-controlled, phase 3 trial. Lancet Oncol. 2019 Feb;20(2):282-96.

6 Abou-Alfa GK, Meyer T, Cheng AL, El-Khoueiry AB, Rimassa L, Ryoo BY, et al. Cabozantinib in patients with advanced and progressing hepatocellular carcinoma. N Engl J Med. 2018 Jul 5;379(1):54-63.

7 Miyahara K, Nouso K, Morimoto Y, Takeuchi Y, Hagihara H, Kuwaki K, et al. Efficacy of sorafenib beyond first progression in patients with metastatic hepatocellular carcinoma. Hepatol Res. 2014 Mar;44(3):296-301.

8 Wada Y, Takami Y, Tateishi M, Ryu T, Mikagi K, Saitsu H. The efficacy of continued sorafenib treatment after radiologic confirmation of progressive disease in patients with advanced hepatocellular carcinoma. PLoS One. 2016;11(1):e0146456.

9 Wada Y, Takami Y, Matsushima H, Tateishi M, Ryu T, Yoshitomi M, et al. Prediction of post-progression survival in patients with advanced hepatocellular carcinoma treated with sorafenib by using time-dependent changes in clinical characteristics. Hepatoma Res. 2018;4(3).

10 Montal R, Andreu-Oller C, Bassaganyas L, Esteban-Fabro R, Moran S, Montironi C, et al. Molecular portrait of high alpha-fetoprotein in hepatocellular carcinoma: implications for biomarker-driven clinical trials. Br J Cancer. 2019 Aug;121(4):340-3.

11 Yada M, Masumoto A, Motomura K, Tajiri H, Morita Y, Suzuki H, et al. Indicators of sorafenib efficacy in patients with advanced hepatocellular carcinoma. World J Gastroenterol. 2014 Sep 21;20(35):12581-7.

12 Casadei Gardini A, Scarpi E, Marisi G, Foschi FG, Donati G, Giampalma E, et al. Early onset of hypertension and serum electrolyte changes as potential predictive factors of activity in advanced HCC patients treated with sorafenib: results from a retrospective analysis of the HCC-AVR group. Oncotarget. 2016 Mar 22;7(12): 15243-51.

13 Howell J, Pinato DJ, Ramaswami R, Bettinger D, Arizumi T, Ferrari C, et al. On-target sorafenib toxicity predicts improved survival in hepatocellular carcinoma: a multi-centre, prospective study. Aliment Pharmacol Ther. 2017 Apr;45(8):1146-55. 